\title{
EVALUACIÓN DE LÍNEAS DE FRIJOL NEGRO, EN VERACRUZ Y CHIAPAS, MÉXICO ${ }^{1}$
}

\author{
Ernesto López ${ }^{2}$, Octavio Cano ${ }^{2}$, Bernardo Villar ${ }^{3}$, Javier Cumpian ${ }^{2}$, Francisco J. Ugalde ${ }^{2}$, Víctor O. López ${ }^{2}$
}

\section{RESUMEN}

Evaluación de líneas de frijol negro, en Veracruz y Chiapas, México. El presente estudio se llevó a cabo durante los ciclos agrícolas de 1999-2000 y 2000-2001; se evaluó la adaptación y rendimiento de 15 líneas avanzadas de frijol negro, así como un testigo local. Dichos genotipos forman parte al Ensayo Centroamericano de Adaptación y Rendimiento (ECAR). La evaluación se realizó en 11 localidades, cinco en el estado de Chiapas y seis en Veracruz, los experimentos se establecieron bajo condiciones de riego, temporal y humedad residual. Las líneas ICTA-Ju 97-1, ICTA-Ju II307, DOR-678 y Cut-45, resultaron sobresalientes por su rendimiento, amplia adaptación y estabilidad. En el ciclo agrícola de otoño-invierno 1999-2000, se evaluó la respuesta de las líneas a las siguientes enfermedades: mosaico dorado (BGMV), mancha angular (Phaseoisariopsis griseola), roya (Uromyces appendiculatus) y la antracnosis (Colletotrichum lindemuthianum). Las líneas que resultaron tolerantes fueron ICTA Ju 97-1 y Cut-45. Ambas serán validadas en campos de agricultores para su posible liberación como nuevas variedades para algunas regiones productoras de Veracruz y Chiapas, México.

\begin{abstract}
Evaluation of bean lines in Veracruz and Chiapas, Mexico. Several experiments were conducted out during the 1999-2000 and 2000-2001 growing cycles: The adaptation and grain yield of 15 advanced lines of black and a local variety used as a control, were evaluated. These genotypes belong to the Central American Assay for Adaptation and Yield (ECAR).The bean genotypes were evaluated in eleven localities, five in the state of Chiapas and six in the state of Veracruz; the experiments were established under irrigated, upland and residual moisture conditions. ICTA Ju 97-1, II307, DOR-678 and Cut-45, were the most oustanding lines for yield, wide range of adaptation and stability. During the 1999-2000 Fall-Winter growing cycle, the response of the lines against Bean Golden Mosaic Virus (BGMV), angular leaf spot (Phaseoisariopsis griseola), rust (Uromyces appendiculatus), and anthracnose (Colletotrichum lindemuthianum) were evaluated; ICTA Ju 97-1 and Cut-45 were tolerant to those diseases. Both lines must be validated in farmer fields in order to be considered for releasing as new varieties for some bean growing areas of Veracruz and Chiapas, Mexico.
\end{abstract}

\section{INTRODUCCION}

El método de mejoramiento por introducción puede ser efectivo para obtener variedades mejoradas, éste consiste en introducir a una localidad o región germoplasma desarrollado en otra región (Miranda 1966), así como la evolución sistemática de materiales importados pueden dar los mismos resultados que los logrados con los métodos de mejoramiento convencionales (CIAT 1986). El Ensayo Centroamericano de Adapta- ción y Rendimiento (ECAR), que es un ensayo de líneas avanzadas empleando en sus evaluaciones un diseño estadístico, pues consiste en probar germoplasma generado en una región y evaluado en diferentes regiones. Este tiene como antecedente al International Bean Yield and Adaptation Nursery (IBYAN) 1979 y al Vivero Centroamericano de Adaptación y Rendimiento (VICAR) 1981. Dichos ensayos han servido para introducir líneas avanzadas de frijol, generadas por el programa de frijol del Centro Internacional de Agricultura

\footnotetext{
1 Recibido para publicación el 5 de abril del 2001. Presentado en la XLVII Reunión Anual del PCCMCA. San José, Costa Rica. 2001.

2 Campo Experimental Cotaxtla. Apartado postal \# 429 Veracruz, Ver., México. Email: salinaser@hotmail.com

3 Campo Experimental Centro de Chiapas. Apartado postal \# 1 Ocozocoautla, Chiapas, México. Email: bervill@prodigy.net.mx
} 
Tropical (CIAT) y el Programa Cooperativo Regional de Frijol para Centroamérica, México y El Caribe (PROFRIJOL), para la región de Centroamérica, México y el Caribe (PROFRIJOL-ICTA 1996). Por medio de las introducciones de materiales por medio de los ECAR, en el sureste mexicano, se identificaron las líneas D-145 y DOR-390,de alto potencial de rendimiento y resistentes a las principales enfermedades de la región. Como resultado de estas acciones en 1981, se libero la variedad Negro Huasteco-81 (Yoshii y Rodríguez 1981), y en 1993, se libero la variedad Negro Tacaná (López et al. 1994a). También la línea DOR-500, próxima a liberarse con el nombre de Negro Tropical (López et al. 1999). Las líneas DOR- 390 y DOR-500, también fueron liberadas en Argentina con los nombres de TUC-390 y TUC-500 (Vizagarra 1997). En otros países de la región se han identificado líneas que después fueron liberadas como nuevas variedades; por ejemplo en Guatemala DOR- 41, DOR-42 DOR-44 y DOR-390 (Soto y Rodríguez 2000); Costa Rica BAT304, BAT- 76 y DOR-60 y Cuba DOR-60, DOR-390, DOR-445, DOR-446 (Voysest, 2000)).

\section{MATERIALES Y MÉTODOS}

Durante el período 1999-2001, se condujeron once ECAR, bajo condiciones de humedad residual, temporal y riego; seis en Veracruz y cinco en Chiapas. Las principales características de los sitios de prueba se presentan en el Cuadro 1. En los ensayos, se incluyó la variedad Negro Jamapa como testigo local, por ser la variedad más sembrada en los estados de Veracruz y Chiapas. En todos los ensayos se utilizó un diseño de bloques completos al azar con tres repeticiones y parcelas de tres surcos de cinco metros de longitud. La fecha de siembra de los experimentos fue de junio para temporal y de septiembre a octubre para humedad residual y riego. Los ensayos se condujeron siguiendo las recomendaciones agronómicas para cada localidad (Nuñez

Cuadro 1. Localización geográfica, temperatura y precipitación media anual en las localidades donde se realizaron los ECAR, en los estados de Veracruz y Chiapas

\begin{tabular}{|c|c|c|c|c|c|}
\hline Localidad & Latitud & Longitud & $\begin{array}{l}\text { Altitud } \\
(\mathbf{m s n m})\end{array}$ & $\begin{array}{c}\text { Temperatura } \\
\left({ }^{\circ} \mathbf{C}\right)\end{array}$ & $\begin{array}{c}\text { Precipitación } \\
(\mathbf{m m})\end{array}$ \\
\hline $\begin{array}{l}\text { Chiapas- } \\
\text { Sur }\end{array}$ & $14^{\circ} 15^{\prime}$ & $92^{\circ} 15^{\prime}$ & 137 & 26,0 & 2488 \\
\hline $\begin{array}{l}\text { Chiapas- } \\
\text { Centro }\end{array}$ & $16^{\circ} 46^{\prime}$ & $93^{\circ} 27^{\prime}$ & 846 & 13,6 & 898 \\
\hline $\begin{array}{l}\text { Veracruz- } \\
\text { Sur }\end{array}$ & $18^{\circ} 06^{\prime}$ & $95^{\circ} 53^{\prime}$ & 25 & 25,0 & 1762 \\
\hline $\begin{array}{l}\text { Veracruz- } \\
\text { Centro }\end{array}$ & $19^{\circ} 12^{\prime}$ & $96^{\circ} 81^{\prime}$ & 14 & 25,5 & 1668 \\
\hline
\end{tabular}

y Carrizales 1984, López et al. 1994b). En todas las localidades se tomaron datos sobre reacción a las enfermedades que ocurrieron durante el ciclo otoño-invierno 1999-2000, la fenolgía de las líneas y el rendimiento. El rendimiento de grano se calculó en $\mathrm{kg} / \mathrm{ha}$ al $14 \%$ de humedad. Los datos del rendimiento se sometieron a un análisis de varianza individual y después se realizó un análisis combinado de los 11 experimentos. Para comparar las medias de tratamientos se utilizó la prueba de Duncan a un nivel de significancia del $0,05 \%$. También se realizó un análisis de regresión con los once experimentos por el método de Eberhart y Russell (1966), y la adaptación y estabilidad de las líneas se calificaron con base al coeficiente y la desviación de la regresión (Carballo y Marquez 1970).

\section{Evaluación de reacción a enfermedades}

Durante la conducción del ECAR del ciclo agrícola 1999-2000, se calificó la reacción de los genotipos evaluados a la incidencia del virus del mosaico dorado, mancha angular, roya y antracnosis; en Tapachula Chiapas (BGMV), Centro y Sur de Veracruz, las demás enfermedades, con una escala de 1 a 9 (CIAT 1987), cuyos valores son: 1a $2=$ resistente, 3 a $4=$ tolerante, 5 a $6=$ medianamente tolerante, 7 a $8=$ medianamente susceptible y $9=$ susceptible. La evaluación de reacción a las enfermedades se realizó durante la etapa reproductiva.

\section{RESULTADOS Y DISCUSIÓN}

\section{Evaluación del rendimiento y adaptación del ECAR}

En la mayoría de los sitios de prueba existieron diferencias altamente significativas entre las líneas estudiadas y en el análisis de conjunto hubo diferencias significativas entre las líneas, localidades y para la interacción línea por localidad. Los genotipos que mostraron los mejores rendimientos en el análisis de conjunto fueron ICTA-Ju 97-1, ICTA-Ju II-307 y Cut-45, los que resultaron estadísticamente iguales entre si, pero diferentes a Negro Jamapa. Las localidades en donde se obtuvieron los mayores rendimientos fueron: Campo Cotaxtla, en el centro de Veracruz, bajo condiciones de riego, en los dos ciclos de prueba OI-99-00 y 00-01. Mientras que los rendimientos más bajos se obtuvieron en Isla, en el Sur de Veracruz, bajo humedad residual. Los bajos rendimientos en esta última localidad fueron debidos a un severo ataque de antracnosis y a la falta de humedad en las etapas reproductivas del cultivo, respectivamente (Cuadro 2). Los resultados 
Cuadro 2. Promedio de rendimiento de 16 genotipos de frijol en 11 ambientes de Veracruz y Chiapas, México, 1999-2001,

\begin{tabular}{lllrl}
\hline \multicolumn{1}{c}{ Localidad } & Ciclo y año & \multicolumn{1}{c}{ Condición } & Rend, kg/ha, \\
\hline Cecot, Veracruz & OI-1999-00 & Riego & $1773 \mathrm{~A}^{1}$ \\
Cecot, Veracruz & OI-2000-01 & Riego & $1346 \mathrm{AB}$ \\
Ocozocoautla, Chiapas & PV-2000 & Temporal & $1153 \mathrm{~B}$ \\
Isla, Veracruz & OI-2000-01 & Humedad Residual & $1060 \mathrm{BC}$ \\
Ocozocoautla, Chiapas & OI-2000-01 & Humedad Residual & $648 \mathrm{CD}$ \\
Ocozocoautla, Chiapas & OI-1999-00 & Humedad Residual & $602 \mathrm{CD}$ \\
Isla, Veracruz & OI-1999-00 & Humedad Residual & $514 \mathrm{D}$ \\
Ocozocoautla, Chiapas & PV-1999 & Temporal & $486 \mathrm{D}$ \\
Tapachula, Chiapas & OI-1999-00 & Riego & $483 \mathrm{D}$ \\
Isla, Veracruz & OI-1999-00 & Humedad Residual & 407 & $\mathrm{D}$ \\
Isla, Veracruz & OI-2000-01 & Humedad Residual & $295 \mathrm{D}$ \\
\hline
\end{tabular}

${ }^{1}$ Prueba de Duncan 0,05

anteriores son similares a los encontrados por López et al. 1994a y López et al. 1999.

Los rendimientos promedios de 11 ambientes, los valores de los parámetros bi y $\mathrm{S}^{2}$ di y la clasificación de cada una de los genotipos con base en el esquema de clasificación propuesto por Carballo y Marquez (1970), se presentan en el Cuadro 3. De los 16 genotipos evaluados para rendimiento en los once ambientes, 13 resultaron estables en su respuesta ( $b i=1 ; S^{2} \mathrm{di}=0$ ); dentro de esta categoría se encontraron las líneas ICTA-Ju 97-1, ICTA-Ju II-307, Cut-45 y Negro Jamapa, junto con nueve líneas experimentales. Dos genotipos: DOR667 e ICTA Ligero se adaptaron mejor en ambientes desfavorables y fueron consistentes $\left(\mathrm{bi}<1 ; \mathrm{S}^{2} \mathrm{di}=0\right)$. Por último, la línea Cut-68, se adaptó mejor en buenos ambientes y fue consistente( $\mathrm{bi}>1 ; \mathrm{S}^{2} \mathrm{di}=0$ ) Los resultados anteriores difieren con los encontrados por López et al. (2000), en donde la línea ICTA-Ju II-307 mostró calificaciones diferentes a las de este trabajo, mientras que los resultados indicados por Profrijol-ICTA (1996), varias de las líneas fueron similares.

\section{Evaluación de reacción a enfermedades}

La enfermedad del virus del mosaico dorado se evaluó en el sur de Chiapas (Tapachula) en el ciclo de Otoño-Invierno 99-00; sembrándose en fecha retrasada, debido a la ocurrencia de fuertes precipitaciones, las que continuaron durante todo el ciclo del cultivo. Las líneas ICTA Ligero e ICTA Ju 97-1, sobresalieron por tener la mejor resistencia a esta enfermedad, así como los grupos DOR e ICTA Ju, los cuales tuvieron buena tolerancia. Las demás enfermedades también fueron evaluadas en el ciclo Otoño-Invierno-99-00, fuera de la fecha óptima de siembra, debido a lo mencionado anteriormente, provocando condiciones ideales para la presencia de enfermedades, tales como la antracnosis en
Cuadro 3. Rendimiento promedio y parámetros de estabilidad de 16 líneas de frijol negro en 11 localidades de Veracruz y Chiapas, México.

\begin{tabular}{lccrc}
\hline Genotipo & Kg/ha & bi & \multicolumn{1}{c}{$\mathbf{S}^{2} \mathbf{d i}$} & Clasificación \\
\hline ICTA Ju 97-1 & 890 & 1,03 & $-7,389,79$ & $\mathrm{~A}$ \\
II-307 & 854 & 1,23 & $-18,403,48$ & $\mathrm{~A}$ \\
DOR-678 & 854 & 0,90 & $-1,039,17$ & $\mathrm{~A}$ \\
Cut-45 & 847 & 1,19 & $-22,233,45$ & $\mathrm{~A}$ \\
DOR-667 & 843 & 0,68 & $-20,566,64$ & $\mathrm{C}$ \\
UCR-55 & 842 & 1,09 & $-27,059,48$ & $\mathrm{~A}$ \\
Cut-68 & 837 & 1,32 & $-11,074,42$ & $\mathrm{E}$ \\
ICTA Ju 91-37 & 822 & 0,85 & $-40,888,65$ & $\mathrm{~A}$ \\
DOR-685 & 808 & 0,93 & $-35,748,83$ & $\mathrm{~A}$ \\
ICTA Ju-95-50 & 793 & 0,97 & $-45,251,76$ & $\mathrm{~A}$ \\
ICTA Ju-93-1 & 793 & 0,84 & $-30,667,35$ & $\mathrm{~A}$ \\
Negro INIFAP & 768 & 1,09 & $-44,259,51$ & $\mathrm{~A}$ \\
Cut-53 & 763 & 1,20 & $-35,516,31$ & $\mathrm{~A}$ \\
Cut-107 & 719 & 0,95 & $-18,979,20$ & $\mathrm{~A}$ \\
Negro Jamapa & 681 & 1,05 & $-19,812,40$ & $\mathrm{~A}$ \\
ICTA Ligero & 637 & 0,69 & $-22,566,82$ & $\mathrm{C}$ \\
PROMEDIO & 797 & & & \\
\hline
\end{tabular}

$\mathrm{A}=$ Estable

$\mathrm{C}=$ Responde mejor en ambientes desfavorables consistente $\mathrm{E}=$ Responde mejor en buenos ambientes consistente

Isla y Cotaxtla en Veracruz, así como la mancha angular. La roya fue evaluada en el centro de Veracruz, las líneas que mostraron mejor resistencia fueron: UCR55, Cut-45 e ICTA Ju 97-1. En este mismo lugar fue evaluada la mancha angular sobresaliendo las líneas y variedades locales: ICTA-Ju II-307, Negro INIFAP y Negro Jamapa. La antracnosis se presentó en Isla, es importante mencionar que las altas precipitaciones y las bajas temperaturas durante la mayor parte del ciclo biológico del cultivo favorecieron el desarrollo de la antracnosis, misma que se identificó con la ayuda de las diferenciales internacionales como la raza 73. Las líneas que presentaron tolerancia a esta enfermedad fueron: UCR-55 y ICTA Ju 93-1 (Cuadro 4). La ocurrencia 
Cuadro 4. Calificación de enfermedades del Ensayo Centro americano de Adaptación y rendimiento, en Veracruz y Chiapas, México. OI-1999-2000.

\begin{tabular}{|c|c|c|c|c|}
\hline Genotipo & BGMV*1 & ROYA*2 & ANTRAC $* * 3$ & MAN ANG*4 \\
\hline ICTA Ju 97-1 & 2,9 & 2,3 & 5,7 & 5,3 \\
\hline II-307 & 5,7 & 4,6 & 8,6 & 2,3 \\
\hline DOR-678 & 3,6 & 5,6 & 6,5 & 6,0 \\
\hline Cut-45 & 5,6 & 2,0 & 5,1 & 4,7 \\
\hline DOR-667 & 3,1 & 3,3 & 6,0 & 6,3 \\
\hline UCR-55 & 6,8 & 2,0 & 4,0 & 4,0 \\
\hline Cut-68 & 6,4 & 5,3 & 9,0 & 7,3 \\
\hline ICTA Ju 91-37 & 3,2 & 6,6 & 7,7 & 7,3 \\
\hline DOR-685 & 3,2 & 5,3 & 6,3 & 4,0 \\
\hline ICTA Ju-95-50 & 3,0 & 3,6 & 7,2 & 5,0 \\
\hline ICTA Ju-93-1 & 4,9 & 3,3 & 4,0 & 7,3 \\
\hline Negro INIFAP & 6,4 & 5,0 & 7,2 & 3,3 \\
\hline Cut-53 & 5,7 & 3,6 & 8,6 & 6,3 \\
\hline Cut-107 & 6,3 & 4,0 & 8,8 & 7,0 \\
\hline Negro Jamapa & - & 7,6 & 8,6 & 3,7 \\
\hline ICTA Ligero & 1,0 & 3,0 & 8,3 & - \\
\hline PROMEDIO & 4,4 & 4,2 & 7,0 & 5,3 \\
\hline $\mathrm{CV} \%$ & 11,1 & 35,5 & 18,9 & 30,2 \\
\hline ANEVA & $* *$ & $*$ & $*$ & $* *$ \\
\hline
\end{tabular}

Enfermedades Escala de 1-9

$*=$ Promedio de tres observaciones

** $=$ Promedio de seis observaciones

$1=$ Tapachula, Chis.

2 = Campo Cotaxtla, Ver.

$3=$ Isla, Ver.

4 = Campo Cotaxtla, Ver.

del fuerte ataque por antracnosis en Isla y Cotaxtla, Veracruz, (datos no incluídos en este trabajo de la última localidad), permitió descubrir el bajo nivel de resistencia a esta enfermedad en el germoplasma mejorado local e introducido a través de PROFRIJOL y se sugiere la necesidad de incorporar resistencia múltiple en forma simultánea a las líneas en desarrollo. En este caso particular se sugiere la utilización de fuentes de resistencia del acervo genético andino.

\section{CONCLUSIONES}

Se identificaron 13 genotipos de frijol estables en rendimiento a través de las localidades de prueba.

Las localidades de mayor rendimiento promedio fueron: Campo Cotaxtla, en el centro de Veracruz y Ocozocoautla, en el centro de Chiapas, bajo condiciones de riego y temporal respectivamente.

Los genotipos que mostraron los mayores rendimientos fueron: ICTA Ju 97-1, II-307 y DOR-678, con rendimientos promedios de 11 ensayos de hasta 890 $\mathrm{kg} / \mathrm{ha}$. El menor rendimiento promedio se observó con ICTA Ligero con $637 \mathrm{~kg} / \mathrm{ha}$.

Las líneas ICTA Ju 97-1, ICTA-Ju II-307 y DOR678 resultaron sobresalientes por su rendimiento, amplia adaptación y estabilidad ( 890, 854 y $854 \mathrm{~kg} / \mathrm{ha}$, bi $=1,0 ; \mathrm{S}^{2} \mathrm{di}=0$ ). Además de presentar resistencia y/o tolerancia a las enfermedades. Con excepción de la antracnosis, la línea ICTA-Ju II-307 resultó tolerante a las enfermedades.

\section{LITERATURA CITADA}

CIAT (CENTRO INTERNACIONAL DE AGRICULTURA TROPICAL). 1986. Mejoramiento de frijol por introducción y selección: unidad audio tuturial sobre el mismo tema. Contenido científico: Voysest, O; López, M. Producción: Fernández, F. Cali, Colombia 32p.

CIAT (CENTRO INTERNACIONAL DE AGRICULTURA TROPICAL). 1987. Sistema estándar para evaluación de germoplasma de frijol. Van Schoonhoven, A.; Pastor, M. eds. Cali, Colombia, CIAT. 56p.

CARBAllo, C. A.; MARQUEZ, F. 1970. Comparación de variedades de maíz del Bajio y de la mesa central por su rendimiento y estabilidad. Agrociencia 5 (1) : 129-146.

EBERHART, S. A.; RUSSELL, W. A. 1966. Stability parameters for comparing varieties. Crop Science $6: 36-40$.

MIRANDA, C. 1966. Mejoramiento del frijol en México. Folleto Miseláneo. Num.13. INIA. SARH. México. 19p.

LÓPEZ, S.E.; BECERRA, E.; CANO, O. 1994a. Reacción al virus del mosaico dorado, adaptación y rendimiento de la línea de frijol DOR-390, en el sureste de México. Revista Mexicana de Fitopatología Vol. 12 (2): 139-145.

LÓPEZ, E.; DURÁN, A.; BECERRA, N.; ESQUEDA, V.; CANO, O.; 1994b. Manual de producción de frijol en el estado de Veracruz. Folleto para productores $\mathrm{N}^{\circ} 7$. México, INIFAP-CIRGOC-CECOT. p. 29.

LÓPEZ, E.; ACOSTA, J.; CANO, O.; FRAIRE, G.; CUMPÍAN, J.; BECERRA, E.; VILLAR, B.; UGALDE, F. 1999. Estabilidad de rendimiento de la línea de frijol negro DOR-500, en el trópico húmedo de México. Agro. Meso.10 (2): 69-74.

LÓPEZ, E.; CUMPÍAN, J.; BECERRA, E.; VILLAR, B.; UGALDE, F.; ACOSTA, J. 2000. Adaptación y rendimiento de la variedad de frijol negro Negro Medellín, en el sureste de México. Agron. Mesoam. 11(2): 47-52. 
NUÑEZ, S.; CARRIZALES, N. 1984. Cómo cultivar frijol en el Centro de Chiapas. Folleto para productores. $\mathrm{N}^{\circ} 4$. México, INIA- CIAPAS. p.15.

PROFRIJOL-ICTA (PROGRAMA COOPERATIVO REGIONAL DE FRIJOL PARA CENTROAMÉRICA, MÉXICO Y EL CARIBE - INSTITUTO DE CIENCIA Y TECNOLOGÍA AGRÍCOLAS). 1996. Informe 19951996. Sistema de viveros y ensayos regionales SISTEVER. Villa Nueva, Guatemala. p.2.

SOTO, J.; RODRÍGUEZ, R. 2000. AMÉRICA CENTRAL, GUATEMALA. In: Morales F. (Ed) El mosaico dorado y otras enfermedades del frijol común causados por geminivirus transmitidos por mosca blanca en América Latina. CIAT. Palmira (Valle), Colombia. p. 25-28.
VIZAGARRA, O. 1997. Tuc-390 y Tuc-500, dos nuevas variedades de poroto negro para el noroeste Argentino. P. 239-246. In: Singh, S. P.; Voysest, O. eds. Taller de mejoramiento para el siglo XXI: Bases para una estrategia para América Latina. CIAT. Calí, Colombia. 559p.

VOYSEST, O. 2000. Mejoramiento genético del frijol (Phaseolus vulgaris L.): Legado de variedades de América Latina 1930-1999. Calí, Colombia, CIAT. 195p.

YOSHII, K.; RODRÍGUEZ, J. 1982. Negro Huasteco-81. Nueva variedad de frijol para elm trópico de México. Instituto de Investigaciónes Agrícolas. México. Folleto Num. 1. 10p. 